\title{
LINEE ISOCLINE RISPETTO ALLE LINEE DI CURVATURA.
}

\author{
Nota di Gustavo Sannia (Torino).
}

Adunanza del 24 novembre 1907 .

I. In questa breve Nota esporró alcuni teoremi, che credo non privi di interesse, sui doppi sistemi di linee di una superficie che in ciascun punto fanno angoli eguali con le linee di curvatúra, e che per breviti chiameró linee isocline. Tali sono, per esempio, le assintotiche nelle regioni a punti iperbolici, le linee caratteristiche (cioe isocline e coniugate) nelle regioni a punti ellittici ${ }^{x}$ ), le linee isocline ed ortogonali o ad immagini sferiche ortogonali ${ }^{2}$ ).

Riferiti i punti di una superficie ad un qualunque doppio sistema di linee coordinate, sieno al solito

$$
\begin{gathered}
d s^{2}=E d u^{2}+2 F d u d v+G d v^{2}, \\
D d u^{2}+2 D^{\prime} d u d v+D^{\prime \prime} d v^{2}, \\
d s^{\prime 2}=e d u^{2}+2 f d u d v+g d v^{2},
\end{gathered}
$$

la $I^{a}$, la $2^{a}$ e la $3^{a}$ forma fondamentale.

E facile ottenere la relazione che lega $\mathrm{i}$ differenziali presi lungo due linee isocline, cioè la condizione affinchè $i$ due elementi lineari $d s$ e $\delta s$, spiccati dal punto $(u, v)$ ai due punti infinitamente vicini $(u+d u, v+d v)$ e $(u+\delta u, v+\delta v)$, facciano angoli eguali con le tangenti alle linee di curvatura.

A tal fine basta osservare che, lungo tali direzioni, la curvatura normale ${ }^{3}$ )

$$
\frac{\mathrm{I}}{R}=-\frac{D d u^{2}+2 D^{\prime} d u d v+D^{\prime \prime} d v^{2}}{E d u^{2}+2 F d u d v+G d v^{2}}
$$

prende valori eguali, per ottenere la relazione cercata

(I) $2\left(D F-E D^{\prime}\right) d u \delta u+\left(D G-E D^{\prime \prime}\right)(d u \delta v+d v \delta u)+2\left(D^{\prime} G-F D^{\prime \prime}\right) d v \delta v=0$.

r) Cfr. : Puccr, Dell'angolo caratteristico e delle linee caratteristiche di una superficie [Rendiconti della R. Accademia dei Lincei, vol. V (1889), Io semestre, pp. 50I-507]; ReINA, Di alcune proprietà delle linee caratteristiche [Ibid., id., PP. 881-885]; EIsENHART, Three particular Systems of lines on a Surface [Transactions of the American Mathematical Society, vol. V (Ig04), Pp. 42I-437].

2) EISENHART, 1. c. I).

3) Branchi, Lezioni di Geometria differenziale, $2^{\mathrm{a}}$ edizione, vol. I, p. I30. 
Ponendo $d=\delta$, si ha l'equazione differenziale delle linee di curvatura

$$
\left(D F-E D^{\prime}\right) d u^{2}+\left(D G-E D^{\prime \prime}\right) d u d v+\left(D^{\prime} G-F D^{\prime \prime}\right) d v^{2}=0 \text {. }
$$

Ponendo invece $d u=0$ e $\delta v=0$, si ha che : affinche le linee coordinate $u=$ cost. e $v=$ cost. sieno isocline, occorre e basta che sia

$$
E D^{\prime \prime}-G D=0 \text {. }
$$

I sistemi di linee isocline sono dunque $i$ soli che riducono a forma canonica il primo membro dell'equazione differenziale delle linee di curvatura.

I coefficienti $e, f, g$ della terza forma fondamentale si esprimono mediante quelli delle altre due con le formole

$$
-e=K E+H D, \quad-f=K F+H D^{\prime}, \quad-g=K G+H D^{\prime \prime},
$$

ove $K$ e $H$ sono la curvatura totale e la curvatura media della superficie, quindi

$$
e D^{\prime \prime}-g D=K\left(G D-E D^{\prime \prime}\right) \text {; }
$$

dunque, escludendo, le superficie sviluppabili $(K=0)$, si puó sostituire alla condizione (2) l'altra

$$
e D^{\prime \prime}-g D=0 \text {. }
$$

Insomma, quando le linee coordinate $u, v$ sono isocline, risulta

$$
E: D: e=G: D^{\prime \prime}: g \text {. }
$$

2. Le derivate seconde delle coordinate $x, y, z$ di un punto mobile di una superficie, espresse in funzione di due parametri $u$ e $v$, si esprimono mediante le derivate prime e mediante i coseni direttivi $X, Y, Z$ della normale alla superficie con le formole ${ }^{4}$ )

$$
\begin{aligned}
\frac{\partial^{2} x}{\partial u^{2}} & =\left\{\begin{array}{c}
\mathrm{II} \\
\mathrm{I}
\end{array}\right\} \frac{\partial x}{\partial u}+\left\{\begin{array}{c}
\mathrm{Ir} \\
2
\end{array}\right\} \frac{\partial x}{\partial v}+D X, \\
\frac{\partial^{2} x}{\partial u \partial v} & =\left\{\begin{array}{c}
\mathrm{I} 2 \\
\mathrm{I}
\end{array}\right\} \frac{\partial x}{\partial u}+\left\{\begin{array}{c}
\mathrm{I} 2 \\
2
\end{array}\right\} \frac{\partial x}{\partial v}+D^{\prime} X, \\
\frac{\partial^{2} x}{\partial v^{2}} & =\left\{\begin{array}{c}
22 \\
\mathrm{I}
\end{array}\right\} \frac{\partial x}{\partial u}+\left\{\begin{array}{c}
22 \\
2
\end{array}\right\} \frac{\partial x}{\partial v}+D^{\prime \prime} X
\end{aligned}
$$

e le analoghe in $y$ e $z$ ove $\mathrm{i}$ simboli di Christoffel $\left\{\begin{array}{c}r s \\ t\end{array}\right\}$ si riferiscono alla prima forma fondamentale.

Supposto che le $u, v$ sieno isocline, e quindi che $E D^{\prime \prime}-G D=0$, ne risulta

$$
G \frac{\partial^{2} x}{\partial u^{2}}-E \frac{\partial^{2} x}{\partial v^{2}}=\left[G\left\{\begin{array}{c}
\mathrm{II} \\
\mathrm{I}
\end{array}\right\}-E\left\{\begin{array}{c}
22 \\
\mathrm{I}
\end{array}\right\}\right] \frac{\partial x}{\partial u}+\left[G\left\{\begin{array}{c}
\mathrm{II} \\
2
\end{array}\right\}-E\left\{\begin{array}{c}
22 \\
2
\end{array}\right\}\right] \frac{\partial x}{\partial u}
$$

con le analoghe in $y$ e $z$.

Posto

$$
p=x^{2}+y^{2}+z^{2}=\sum x^{2},
$$

4) BiANCHI, 1. c. 3), pag. I 6 . 
si ha

(3)

$$
\begin{gathered}
\frac{\partial \rho}{\partial u}=2 \sum x \frac{\partial x}{\partial u}, \quad \frac{\partial \rho}{\partial v}=2 \sum x \frac{\partial x}{\partial v} \\
\left\{\begin{array}{l}
\frac{\partial^{2} p}{\partial u^{2}}=2 \sum\left(\frac{\partial x}{\partial u}\right)^{2}+2 \sum x \frac{\partial^{2} x}{\partial u^{2}}=2 E+\left\{\begin{array}{c}
\mathrm{II} \\
\mathrm{I}
\end{array}\right\} \frac{\partial \rho}{\partial u}+\left\{\begin{array}{c}
\mathrm{II} \\
2
\end{array}\right\} \frac{\partial \rho}{\partial v}+D \sum x X \\
\frac{\partial^{2} \rho}{\partial v^{2}}=2 \sum\left(\frac{\partial x}{\partial v}\right)^{2}+2 \sum x \frac{\partial^{2} x}{\partial v^{2}}=2 G+\left\{\begin{array}{c}
22 \\
I
\end{array}\right\} \frac{\partial p}{\partial u}+\left\{\begin{array}{c}
22 \\
2
\end{array}\right\} \frac{\partial \rho}{\partial v}+D^{\prime \prime} \sum x X
\end{array}\right.
\end{gathered}
$$

quindi

$$
G \frac{\partial^{2} p}{\partial u^{2}}-E \frac{\partial^{2} p}{\partial v^{2}}=\left[G\left\{\begin{array}{c}
I I \\
I
\end{array}\right\}-E\left\{\begin{array}{c}
22 \\
I
\end{array}\right\}\right] \frac{\partial p}{\partial u}+\left[G\left\{\begin{array}{c}
I I \\
2
\end{array}\right\}-E\left\{\begin{array}{c}
22 \\
2
\end{array}\right\}\right] \frac{\partial p}{\partial v} .
$$

Dunque: Le coordinate cartesiane ortogonali $x, y, z$ di un punto mobile di una superficie, espresse in funzione dei parametri $u, v$ di due sistemi di linee isocline, $e p=x^{2}+y^{2}+z^{2}$ sono soluzioni di una medesima equazione lineare del tipo

$$
a \frac{\partial^{2} \varphi}{\partial u^{2}}-b \frac{\partial^{2} \varphi}{\partial v^{2}}=x \frac{\partial \varphi}{\partial u}+\beta \frac{\partial \varphi}{\partial v}
$$

ove $a$ e $b$ sono proporzionali ai coefficienti estremi delle tre forme fondamentali.

Viceversa: supponiamo che $x, y, z \rho$ sieno quattro soluzioni particolari di una medesima equazione del tipo (4). Scrivendo che la (4) è soddisfatta da $x$, y e $z$ moitiplicando per $2 x, 2 y, 2 z$ le equazioni che ne risultano e poi sommandole, si ha per le (3)

e quindi

$$
a\left(\frac{\partial^{2} p}{\partial u^{2}}-2 E\right)-b\left(\frac{\partial^{2} p}{\partial v^{2}}-2 G\right)=\alpha \frac{\partial \rho}{\partial u}+\beta \frac{\partial \rho}{\partial v}
$$

$$
a E-b G=0 \text {, }
$$

essendo anche $\rho$ una soluzione della (4).

Moltiplicando invece le stesse equazioni per $X, Y, Z$ e poi sommandole, si ha

ossia

$$
a \sum X \frac{\partial^{2} x}{\partial u^{2}}-b \sum X \frac{\partial^{2} x}{\partial v^{2}}=x \sum X \frac{\partial x}{\partial u}+\beta \sum X \frac{\partial x}{\partial v}=0,
$$

$$
a D-b D^{\prime \prime}=0 \text {. }
$$

Dalle (5) e (6) risulta $E D^{\prime \prime}-G D=$ o.

Dunque viceversa: se le coordinate $x(u, v), y(u, v), z(u, v)$ e $p=x^{2}+y^{2}+z^{2}$ sono soluzioni di una medesima equazione del tipo (4), sulla superficie

$$
x=x(u, v), \quad y=y(u, v), \quad z=z(u, v)
$$

le linee $u, v$ sono isocline. Si ha inoltre

$$
E: D: e: a=G: D^{\prime \prime}: g: b \text {. }
$$

3. Si puo osservare che: $x, y, z \rho$ non possono soddisfare due equazioni distinte del tipo (4).

Si supponga infatti che $x, y, z \rho$ soddisfino la (4) e l'altra

$$
a^{\prime} \frac{\partial^{2} \varphi}{\partial u^{2}}-b^{\prime} \frac{\partial^{2} \varphi}{\partial v^{2}}=\alpha^{\prime} \frac{\partial \varphi}{\partial u}+\beta^{\prime} \frac{\partial \varphi}{\partial v} \text {. }
$$


Risulta dalla dimostrazione del teorema precedente che $a E-b G=0$ e $a^{\prime} E-b^{\prime} G=0$, quindi $\frac{a^{\prime}}{a}=\frac{b^{\prime}}{b}=k$ ed allora $x, y, z, \rho$ soddisferanno anche l'equazione

$$
\left(\alpha^{\prime}-k \alpha\right) \frac{\partial \varphi}{\partial u}+\left(\beta^{\prime}-k \beta\right) \frac{\partial \varphi}{\partial v}=0 .
$$

Scrivendo che questa è soddisfatta da $x, y$, $z$ moltiplicando le equazioni che ne risultano una volta per $\frac{\partial x}{\partial u}, \frac{\partial y}{\partial u}, \frac{\partial z}{\partial u}$ ed un'altra per $\frac{\partial x}{\partial v}, \frac{\partial y}{\partial v}, \frac{\partial z}{\partial v}$ e poi sommandole, si ha:

$$
\begin{aligned}
& \left(\alpha^{\prime}-k \alpha\right) E+\left(\beta^{\prime}-k \beta\right) F=0, \\
& \left(\alpha^{\prime}-k \alpha\right) F+\left(\beta^{\prime}-k \beta\right) G=0,
\end{aligned}
$$

quindi $\alpha^{\prime}-k \alpha=0, \beta^{\prime}-k \beta=0$, essendo $E G-F^{2}>0$. Dunque

$$
\frac{a^{\prime}}{a}=\frac{b^{\prime}}{b}=\frac{\alpha^{\prime}}{\alpha}=\frac{\beta^{\prime}}{\beta} \text {, }
$$

cioè le due equazioni cui soddisfanno $x, y, z, p$ coincidono.

4. Per mezzo dei teoremi precedenti si puo dimostrare facilmente che: l'inversione per raggi vettori reciproci conserva $i$ sistemi di linee isocline (cio che d'altronde è evidente poiche l'inversione conserva gli angoli e le linee di curvatura).

Le formole per l'inversione, rispetto alla sfera che ha per centro l'origine o e per raggio $R$, sono

$$
x^{\prime}=\frac{R^{2} x}{x^{2}+y^{2}+z^{2}}, \quad y^{\prime}=\frac{R^{2} y}{x^{2}+y^{2}+z^{2}}, \quad z^{\prime}=\frac{R^{2} z}{x^{2}+y^{2}+z^{2}} .
$$

Supposto che le $u, v$ sieno linee isocline, $x, y, z, p$ sono soluzioni di una medesima equazione del tipo (4). Or posto

$$
\varphi^{\prime}=\frac{R^{2} \varphi}{\rho},
$$

La (4) si cambia in una equazione dello stesso tipo, come è facile verificare, e ciò perchè $\rho$ è un integrale della (4). La nuova equazione avrà per integrali particolari

$$
\frac{R^{2} x}{\rho}=x^{\prime}, \quad \frac{R^{2} y}{\rho}=y^{\prime}, \quad \frac{R^{2} z}{\rho}=z^{\prime}, \quad \frac{R^{2} \cdot R^{2}}{\rho}=x^{\prime 2}+y^{\prime 2}+z^{\prime 2},
$$

quindi sulla superficie trasformata, luogo del punto $\left(x^{\prime}, y^{\prime}, z^{\prime}\right)$, le $u, v$ sono linee isocline.

5. E noto che, affinchè sulla superficie

$$
x=x(u, v), \quad y=y(u, v), \quad z=z(u, v)
$$

le linee $u, v$ formino un doppio sistema coniugato, occorre e basta che $x, y, z$ sieno soluzione di una medesima equazione di LAPLACE

$$
\frac{\partial^{2} \varphi}{\partial u \partial v}=A \frac{\partial \varphi}{\partial u}+B \frac{\partial \varphi}{\partial v} \text {. }
$$

Combinando questo teorema con quelli del $\ 2$ si ha: affinche sulla superficie (7) le $u, v$ sieno le linee caratteristiche occorre e basta che $x, y$, $z$ soddisfino contemporaneamente a due equazioni del tipo (4) $e\left(7^{*}\right)$ e che alla prima soddisfi anche $p=x^{2}+y^{2}+z^{2}$. 
Un teorema analogo per le linee isocline ed ortogonali si puó ottenere combinando $\mathrm{i}$ teoremi del $\ 2$ col seguente:

Le coordinate $x, y, z$ di un punto mobile di una superficie (che non sia un cono col vertice nell'origine) soddisfanno una medesima equazione del tipo

$$
\frac{\partial^{2} \varphi}{\partial u \partial v}=A \frac{\partial \varphi}{\partial u}+B \frac{\partial \varphi}{\partial v}+C \varphi \text {. }
$$

Affinche le $u$, $v$ sieno ortogonali occorre e basta che $p=x^{2}+y^{2}+z^{2}$ soddisf l'equazione

$$
\frac{\partial^{2} \varphi}{\partial u \partial v}=A \frac{\partial \varphi}{\partial u}+B \frac{\partial \varphi}{\partial v}+2 C \varphi \text {. }
$$

Infatti, scrivendo che la (8) è soddisfatta da $x, y, z$ si ha un sistema di equazioni lineari in $A, B, C$ che determinano $A, B, C$ in un sol modo, perchè il determinante dei coefficienti

non è nullo. Invero ${ }^{5}$ )

$$
\Delta=\left|\begin{array}{lll}
\frac{\partial x}{\partial u} & \frac{\partial x}{\partial v} & x \\
\frac{\partial y}{\partial u} & \frac{\partial y}{\partial v} & y \\
\frac{\partial z}{\partial u} & \frac{\partial z}{\partial v} & z
\end{array}\right|
$$

e moltiplicando per verticali

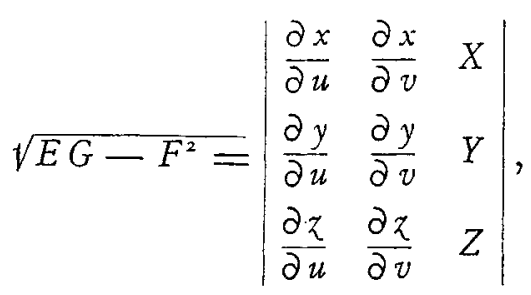

$$
\Delta \cdot \sqrt{E G-F^{2}}=\left|\begin{array}{ccc}
E & F & 0 \\
F & G & 0 \\
\sum x \frac{\partial x}{\partial u} & \sum x \frac{\partial x}{\partial v} & \sum x X
\end{array}\right|=\left(E G-F^{2}\right) \sum x X,
$$

da cui

$$
\Delta=\sqrt{E G-F^{2}} \cdot \sum x X
$$

Ora $\sum x X$ è la distanza del piano tangente alla superficie nel punto $(x, y, z)$ dall'origine, e non è nulla, se la superficie non è un cono col vertice nell'origine; dunque $\Delta$ non è nullo.

Si ha inoltre

5) BIANCHI, 1. c. 3), pag. II5. 


$$
\begin{aligned}
\frac{\partial p}{\partial u}=2 \sum x \frac{\partial x}{\partial u} \\
\frac{\partial^{2} p}{\partial u \partial v}=2 \sum \frac{\partial x}{\partial v} \frac{\partial x}{\partial u}+2 \sum x \frac{\partial^{2} x}{\partial u \partial v} \\
=2 F+2 \sum x\left(A \frac{\partial x}{\partial u}+B \frac{\partial x}{\partial v}+C x\right) \\
=2 F+A \frac{\partial p}{\partial u}+B \frac{\partial p}{\partial v}+2 C p ;
\end{aligned}
$$

ne segue che, affinchè le $u, v$ sieno ortogonali, ossia affinchè risulti $F=0$, occorre e basta che $\rho$ soddisfi la (9).

6. Le derivate seconde dei coseni direttivi $X, Y, Z$ della normale ad una superficie si esprimono mediante $\mathrm{i}$ coseni stessi e le loto derivate prime con le formole ${ }^{6}$ )

$$
\begin{aligned}
\frac{\partial^{2} X}{\partial u^{2}} & =\left\{\begin{array}{l}
\text { II } \\
I
\end{array}\right\} \frac{\partial X}{\partial u}+\left\{\begin{array}{c}
\text { II } \\
2
\end{array}\right\} \frac{\partial X}{\partial v}-e X, \\
\frac{\partial^{2} X}{\partial u \partial v} & =\left\{\begin{array}{c}
\text { I } 2 \\
\mathrm{I}
\end{array}\right\} \frac{\partial X}{\partial u}+\left\{\begin{array}{c}
\mathrm{I} 2 \\
2
\end{array}\right\} \frac{\partial X}{\partial v}-f X, \\
\frac{\partial^{2} X}{\partial v^{2}} & =\left\{\begin{array}{c}
22 \\
\mathrm{I}
\end{array}\right\} \frac{\partial X}{\partial u}+\left\{\begin{array}{c}
22 \\
2
\end{array}\right\} \frac{\partial X}{\partial v}-g X
\end{aligned}
$$

e le analoghe in $Y$ e $Z$, ove $i$ simboli di Christofrel $\left\{\begin{array}{c}r s i \\ t\end{array}\right\}$ si riferiscono alla terza forma fondamentale.

$\mathrm{Ne}$ risulta

$$
\left.\left.g \frac{\partial^{2} X}{\partial u^{2}}-e \frac{\partial^{2} X}{\partial v^{2}}=\left[\begin{array}{c}
g \\
\text { II I I }
\end{array}\right\}-e\left\{\begin{array}{c}
22 \\
\mathrm{I}
\end{array}\right\}\right] \frac{\partial X}{\partial u}+\left[\begin{array}{c}
g(\mathrm{II} \\
2
\end{array}\right\}-e\left\{\begin{array}{c}
22 \\
2
\end{array}\right\}\right] \frac{\partial X}{\partial v},
$$

con le analoghe in $Y$ e $Z$.

Detta $W$ la distanza del piano tangente alla superficie nel punto $(x, y, z)$ dall'origine, cioè posto

si ha ${ }^{7}$ )

$$
W=x X+y Y+z Z
$$

$$
\begin{aligned}
& \frac{\partial^{2} W}{\partial u^{2}}-\left\{\begin{array}{c}
\mathrm{II} \\
\mathrm{I}
\end{array}\right\} \frac{\partial W}{\partial u}-\left\{\begin{array}{c}
\mathrm{II} \\
2
\end{array}\right\} \frac{\partial W}{\partial v}+e W=-D, \\
& \frac{\partial^{2} W}{\partial v^{2}}-\left\{\begin{array}{c}
22 \\
\mathrm{I}
\end{array}\right\} \frac{\partial W}{\partial u}-\left\{\begin{array}{c}
22 \\
2
\end{array}\right\} \frac{\partial W}{\partial v}+g W=-D^{\prime \prime}
\end{aligned}
$$

quindi, se le $u, v$ sono isocline, cioè se $e D^{\prime \prime}-g D=0$, si ha

$$
g \frac{\partial^{2} W}{\partial u^{2}}-e \frac{\partial^{2} W}{\partial v^{2}}=\left[g\left\{\begin{array}{c}
\text { II } \\
\mathrm{I}
\end{array}\right\}-e\left\{\begin{array}{c}
22 \\
\mathrm{I}
\end{array}\right\}\right] \frac{\partial W}{\partial u}+\left[g\left\{\begin{array}{c}
\mathrm{II} \\
2
\end{array}\right\}-e\left\{\begin{array}{c}
22 \\
2
\end{array}\right\}\right] \frac{\partial W}{\partial v} .
$$

Dunque: le coordinate tangenziali $X, Y, Z, W$ di un punto mobile di una superficie, espresse in funzione dei parametri $u, v d i$ due sistemi di linee isocline, sono soluzioni di una medesima equazione del tipo (4), ove $a$ e $b$ sono proporzionali ai coefficienti estremi delle tre forme fondamentali.

6) BIANCHI, 1. c. 3), pag. Is2.

7) Bianchi, 1. c. 3), pag. I73. 
Viceversa: supponiamo che le coordinate tangenziali $X, Y, Z, W$ soddisfino una medesima equazione del tipo (4).

Essendo ${ }^{8}$ )

risulta

$$
D=\sum x \frac{\partial^{2} X}{\partial u^{2}}-\frac{\partial^{2} W}{\partial u^{2}}, \quad D^{\prime \prime}=\sum x \frac{\partial^{2} X}{\partial v^{2}}-\frac{\partial^{2} W}{\partial v^{2}}
$$

$$
\begin{aligned}
a D-b D^{\prime \prime} & =\sum x\left(a \frac{\partial^{2} X}{\partial u^{2}}-b \frac{\partial^{2} X}{\partial v^{2}}\right)-\left(a \frac{\partial^{2} W}{\partial u^{2}}-b \frac{\partial^{2} W}{\partial v^{2}}\right) \\
& =\sum x\left(\alpha \frac{\partial X}{\partial u}+\beta \frac{\partial X}{\partial v}\right)-\left(\alpha \frac{\partial W}{\partial u}+\beta \frac{\partial W}{\partial v}\right) \\
& =\alpha\left(\sum x \frac{\partial X}{\partial u}-\frac{\partial W}{\partial u}\right)+\beta\left(\sum x \frac{\partial X}{\partial v}-\frac{\partial W}{\partial v}\right) \\
& =-\alpha \sum X \frac{\partial x}{\partial u}-\beta \sum X \frac{\partial x}{\partial v}=0
\end{aligned}
$$

poi derivando la $X^{2}+Y^{2}+Z^{2}=\mathrm{r}$, si ha:

quindi

$$
\sum X \frac{\partial^{2} X}{\partial u^{2}}+e=0, \quad \sum X \frac{\partial^{2} X}{\partial v^{2}}+g=0
$$

$$
a e-b g=-\sum X\left(a \frac{\partial^{2} X}{\partial u^{2}}-b \frac{\partial^{2} X}{\partial v^{2}}\right)=-\sum X\left(\alpha \frac{\partial X}{\partial u}+\beta \frac{\partial X}{\partial v}\right)=0 .
$$

$\mathrm{Da} a D-b D^{\prime \prime}=\mathrm{o}$ ed $a e-b g=\mathrm{o}$ segue $e D^{\prime \prime}-g D=0$; dunque: se le coordinate tangenziali $X, Y, Z, W$ di un punto mobile di una superficie, espresse in funzione di due parametri $u, v$, soddisfanno ad una medesina equazione del tipo (4), le $u, v$ segnano sulla superficie due sistemi di linee isocline. I coefficienti $a$ e $b$ della (4) sono proporzionali ai coefficienti estremi delle tre forme fondamentali.

E facile vedere, come al $\ 3$, che $X, Y, Z, W$ non possono soddisfare a due equazioni distinte del tipo (4):

7. I teoremi dei $\$ \ 2$ e 6 si possono generalizzare.

Se di una equazione lineare del tipo

$$
a \frac{\partial^{2} \varphi}{\partial u^{2}}-b \frac{\partial^{2} \varphi}{\partial v^{2}}=\alpha \frac{\partial \varphi}{\partial u}+\beta \frac{\partial \varphi}{\partial v}+\gamma \varphi
$$

si conoscono cinque integrali particolari legati da una relazione del secondo grado ed omogenea a coefficienti costanti, si possono facilmente costruire superficie su cui le $u$, $v$ sono linee isocline.

Sieno $\hat{\imath}_{1}, p_{2}, p_{i}, \hat{p}_{4}, \varphi_{5}$ cinque integrali particolari della (Io) legati dalla relazione

$$
\Phi\left(\varphi_{1}, \varphi_{2}, \varphi_{3}, \varphi_{4}, \varphi_{5}\right)=0,
$$

ove $\Phi$ è una forma quadratica a coefficienti costanti.

8) BIANCHI, 1. c. 3), pag. 173 .

Rend. Circ. Maters. Palermo, t. XXV ( $\left.{ }^{\circ} \mathrm{sem} .1908\right) .-$ Stampato il 21 gennajo 1908. 
Se sulle (II) si esegua la sostituzione lineare omogenea

$$
\psi_{i}=c_{i 1} \varphi_{1}+c_{i 2} \varphi_{2}+\cdots+c_{i s} \varphi_{s}
$$$$
(i=1,2, \ldots, 5)
$$

a coefficienti $c_{i j}$ costanti, si ha una nuova relazione quadratica

$$
\Psi\left(\psi_{1}, \psi_{2}, \psi_{3}, \psi_{4}, \psi_{5}\right)=0
$$

ove $\psi_{1}, \psi_{2}, \ldots, \psi_{5}$ sono pure integrali particolari della (ro).

I. Si possono sempre determinare i coefficienti $c_{i j}$ della sostituzione in modo che la (12) si tiduca a

$$
\psi_{1}^{2}+\psi_{2}^{2}+\psi_{3}^{2}-\psi_{4} \psi_{5}=0 \text {. }
$$

Posto $\varphi=\psi_{5} \cdot \psi_{,}$, la (ro) si trasforma in un'altra equazione dello stesso tipo in $\psi$, ma che ammetterd l'integrale $\psi=\psi_{5}: \psi_{s}=$ I, e peró sard del tipo (4). Essa ammetterd per integrali

$$
\begin{gathered}
x=\frac{\psi_{1}}{\psi_{5}}, \quad y=\frac{\psi_{2}}{\psi_{s}}, \quad z=\frac{\psi_{3}}{\psi_{5}}, \\
\beta=\frac{\psi_{4}}{\psi_{s}}=\frac{\psi_{s}^{2}}{\psi_{5}^{2}}+\frac{\psi_{2}^{2}}{\psi_{5}^{2}}+\frac{\psi_{3}^{2}}{\psi_{5}^{2}}=x^{2}+y^{2}+z^{2},
\end{gathered}
$$

quindi sulla superficie descritta dal punto $(x, y, z)$, le linee $u, v$ saranno isocline.

II. Si possono sempre determinare le costanti $c_{i j}$ in modo che la (12) si riduca a

$$
\psi_{1}^{2}+\psi_{2}^{2}+\psi_{13}^{2}-\psi_{4}^{2}=0
$$

Con la sostituzione $\varphi=\psi_{4} \cdot \psi$, la (10) si trasformerd in un'altra del tipo (4), perchè ammetterd l'integrale $\psi=\psi_{4}: \psi_{4}=\mathrm{I}$; che avrà per integrali

$$
X=\frac{\psi_{1}}{\psi_{4}}, \quad Y=\frac{\psi_{2}}{\psi_{4}}, \quad Z=\frac{\psi_{3}}{\psi_{4}}, \quad W=\frac{\psi_{5}}{\psi_{4}}
$$

e fra i primi tre passerd la relazione

$$
X^{2}+Y^{2}+Z^{2}=\mathbf{x}
$$

Dunque, sulla superficie luogo del punto di coordinate tangenziali $X, Y, Z, W$, le linee $u, v$ saranno isocline.

Napoli, 29 luglio 1907.

Gustavo Sania. 